\title{
MicroRNA-150 inhibits the proliferation and metastasis potential of colorectal cancer cells by targeting iASPP
}

\author{
CHEN LI, XIAOHUI DU, SHAOYOU XIA and LIN CHEN \\ Department of Surgery, Clinical Division, The Chinese People's Liberation \\ Army General Hospital, Beijing 100853, P.R. China
}

Received August 14, 2017; Accepted April 19, 2018

DOI: $10.3892 /$ or.2018.6406

\begin{abstract}
In the present study, the function of $m i R-150$ and its downstream target iASPP in the growth and metastasis of colorectal cancer (CRC) cells was investigated. The expression of $m i R-150$ and iASPP was first investigated in clinical CRC samples. Subsequently, the effects of $m i R-150$ overexpression and iASPP inhibition on cell viability, cell cycle distribution, apoptosis, migration and invasion were detected with CCK-8, flow cytometry, scratch and Transwell assays. The interaction between $m i R$ - 150 and iASPP was confirmed using a dual-luciferase assay. Subsequently, the key role of iASPP in the anti-CRC function of $m i R-150$ was assessed by inducing the expression of the gene in $m i R-150$ overexpressed SW480 cells. In clinical samples, the level of $m i R-150$ was downregulated, while iASPP was induced. Enforced expression of $m i R-150$ decreased the viability, induced G1 cell cycle arrest and apoptosis, and inhibited the migration and invasion of SW480 cells. Knockdown of $i A S P P$ exerted a similar effect on SW480 cells to that of the overexpression of $m i R-150$. Dual-luciferase assay demonstrated that $m i R-150$ directly bound to $i A S P P$ and inhibited its transcription. The function of $m i R-150$ depended on the inhibition of iASPP; induced expression of iASPP in $m i R-150$-knockdown SW480 and HCT116 cells restored cell viability, migration and invasion while inhibiting G1 cell cycle arrest and apoptosis. Increased expression of $m i R-150$ suppressed viability, proliferation, migration and invasion of SW480 cells. Furthermore, iASPP was a direct target of $m i R-150$ and played a key role in its anti-CRC function. $m i R-150$ may be a promising predictor of prognosis in CRC patients.
\end{abstract}

\section{Introduction}

Colorectal cancer (CRC) remains the fourth most common cause of cancer-related deaths worldwide (1), with more than

Correspondence to: Professor Xiaohui Du, Department of Surgery, Clinical Division, The Chinese People's Liberation Army General Hospital, 28 Fuxing Road, Haidian, Beijing 100853, P.R. China E-mail:dr.duxh@hotmail.com

Key words: colorectal cancer, iASPP, invasion, microRNA-150, migration
1.2 million new cases being identified annually (2). Although most cases of CRC are sporadic, 20-30\% of individuals with CRC carry inherited mutations in key tumor suppressors, such as APC and TP53 $(3,4)$. Compared with other solid malignancies, CRC is characterized by slow development, which renders the tumor curable and preventable. Additionally, the survival rate of patients with CRC is critically dependent on the tumor stage at diagnosis. Thus, early diagnosis of CRC has become a central subject in the field of CRC studies. The identification of robust molecular indicators associated with the proliferation and tumor-node-metastasis (TNM) potential of CRC can lead to avoiding understaging of the tumor and help to pinpoint patients with early-stage CRC. In recent years, studies regarding microRNA (miR) have indicated the potential of this non-coding RNA type in categorizing the subtype and prognosis of CRC (5-7).

miR can suppress mRNA translation of targeted genes by binding to the $3^{\prime}$ untranslated region (UTR) of target messenger RNA (mRNA) (8). Dysregulation of miRs has been demonstrated to be associated with tumorigenesis of various human organs, including the colorectum $(9,10)$. Liu et al $(6)$ revealed that miR-124 upregulation reduced cell viability and proliferation of CRC cells in vitro. In another clinical study, Sarlinova et al (11) reported significantly upregulated expression of $m i R-21$ and $m i R-221$ and downregulation of $m i R-150$ in blood samples of CRC patients. As one of the most studied miRs in various types of cancer (12-14), the positive correlation between the expression of $m i R-150$ and the survival of CRC patients has been long revealed $(15,16)$. Furthermore, the dysregulation of $m i R-150$ in CRC was subsequently studied by Wang et al (7) and Feng et al (17), who revealed the antagonistic effect of miR-150 on the oncogenesis and progression of CRC via targeting MUC4 and c-Myb. Given that the above-mentioned studies markedly indicated that $m i R-150$ is a tumor suppressor gene in CRC, it is reasonable to further explore the mechanism driving the antitumor function of $m i R-150$ in CRC.

Inhibitor of apoptosis stimulating protein of p53 (iASPP) belongs to the ASPP family (18). This factor can inhibit the normal function of p53, which leads to oncogenesis in human organs $(19,20)$. Furthermore, iASPP can also negatively regulate the p65 subunit of nuclear factor- $\kappa \mathrm{B}(\mathrm{NF}-\kappa \mathrm{B})$, which plays a vital function in inflammation and apoptosis (21). Therefore, suppressing the function of iASPP may serve as a promising 
therapeutic strategy for the prevention and treatment of CRC. Based on bioinformatic analysis, iASPP is a potential target of $m i R-150$ and regulation of iASPP by $m i R-150$ may influence the biological features of CRC cells. To verify our hypothesis in the present study, we challenged the expression of $m i R-150$ in clinical samples and then detected the effect of $m i R-150$ induction/iASPP inhibition on the viability, apoptosis and mobility of CRC cells. The findings outlined in the present study confirmed the direct regulation of iASPP by $m i R-150$, which would impair the growth and metastasis potential of CRC.

\section{Materials and methods}

Reagents and chemicals. Antibodies against iASPP (cat. no. ab115605) and GAPDH (cat. no. KC-5G5) were purchased from Abcam (Cambridge, UK) and Kangcheng Bio (Beijing, China), respectively. Hoechst staining kit (cat. no. H1399) was purchased from Thermo Fisher Scientific (Waltham, MA, USA). IgG-HRP antibody (cat. no. BA1054) was purchased from Wuhan Boster Biological Technology Ltd. (Wuhan, China).

Patient and CRC specimen collection. CRC specimens were collected from 30 patients (from July 2015 to October 2016) in The Chinese People's Liberation Army General Hospital. The specimens were fixed and prepared in paraffin sections. The patients enrolled in the present study were diagnosed with primary CRC and had detailed clinicopathological and prognostic information. Screening, inspection and data collection were approved by the Ethics Committee of The Chinese People's Liberation Army General Hospital, and a written informed consent form was signed by all subjects. The procedures performed adhered to the Declaration of Helsinki.

Immunohistochemistry. Immunohistochemistry was used to detect the expression of iASPP protein in tissues, according to the operation guide of immunohistochemistry. Paraformaldehyde (4\%) fixed tissues and paraffin embedded sections for antigen retrieval were used. Samples were then incubated overnight at $4^{\circ} \mathrm{C}$ with iASPP antibodies (1:400). After being washed with PBS, samples were incubated for $50 \mathrm{~min}$ at RT with IgG-HRP antibodies (1:500). Then the samples were observed and images were captured by an optical microscope (CX41-23C02; Olympus Corp., Tokyo, Japan).

Cell culture. Human CRC cell lines FHC, HCT116, HCT8, HT29, H1299 and SW480 were obtained from Shanghai Bioleaf Biotech, Co., Ltd., (Shanghai, China). The cells were cultured in minimum essential medium (MEM, M2279; Sigma-Aldrich, St. Louis, MO, USA) with $15 \%$ fetal bovine serum (FBS; 10099-141; Gibco, Carlsbad, CA, USA) and 1\% (v/v) antibiotics mix and maintained in an atmosphere of $95 \%$ air and $5 \% \mathrm{CO}_{2}$ at $37^{\circ} \mathrm{C}$. The expression level of $m i R-150$ was determined using reverse transcription real-time PCR (qPCR) as described in the following sections. The cell line with the lowest expression level of $m i R-150$ was selected for subsequent assays and, based on the results of qPCR, SW480 cell line had the lowest expression level of $m i R-150$ (Fig. 1D) and was employed as an in vitro model for CRC.
Construction of vector, sequences of siRNA and transfection. Specific siRNA targeting iASPP (5'-AGTTCATGTC CAGAAAGTCCC-3') and non-targeting siRNA (5'-ACGU GACACGUUCGGAGAATT-3') were used to knockdown the expression of iASPP. Coding sequences were cloned through amplification reaction using primers (iASPP forward, 5'-GGGGTACCATGGACAGCGAGGCATTCC-3' and iASPP reverse, 5'-CCGCTCGAGCTAGACTTTACTCCTTTGAG GCTTCAC-3'). Subsequently, the PCR product (2487 bp) was ligated to the pcDNA3.0 plasmid, and recombinant plasmid was confirmed by sequencing after digestion with KpnI/XhoI. SW480 cells were transfected with different vectors using Lipofectamine 2000 (Invitrogen; Thermo Fisher Scientific). The coding sequence of $i A S P P$ was ligated into the pcDNA plasmid to form the pcDNA-iASPP vector for overexpression of the gene.

Experimental design and grouping. To detect the function of $m i R-150$ in the oncogenesis of CRC, SW480 cells were divided into two groups: i) NC group, SW480 cells transfected with NC mimics; and ii) mimics group, SW480 cells transfected with $m i R-150$ mimics. Each group was represented by at least five replicates.

To elucidate the key role of iASPP in the progression of CRC, SW480 cells were divided into three groups: i) Blank group, SW480 cells; ii) NC group, SW480 cells transfected with pcDNA-NC plasmid; and iii) siRNA group, SW480 cells transfected with pcDNA-siiASPP plasmid. Each group was represented by at least five replicates.

The interaction between miR-150 and iASPP was further assessed with four groups: i) blank group, SW480 cells; ii) NC group, SW480 cells transfected with NC mimics; iii) mimics group, SW480 cells transfected with $m i R-150$ mimics; and iv) mimics+pcDNA group, $m i R-150$ stably overexpressed in SW480 cells transfected with pcDNA-siiASPP plasmid.

Dual-Luciferase assay. The direct regulating function of $m i R-150$ on the 3'UTR of $i A S P P$ was determined with a Dual-Luciferase assay. Luciferase activity was detected by Dual-Luciferase assay kit (E1960; Promega, Madison, WI, USA) after $24 \mathrm{~h}$ of transfection and co-transfection of Renilla luciferase plasmid, used as the internal control for transfection efficiency.

Real-time PCR. Total RNA in the cells was extracted by RNA purification using RNA Extraction kit (9109; Takara Bio, Inc., Otsu, Japan) accordingly. $\beta$-actin and U6 were selected as the internal reference genes. cDNA templates were achieved by using Super MMLV Reverse Transcriptase (DBI-2342; DBI Bioscience, Shanghai, China), and the final RT-qPCR reaction mix contained $10 \mu 1$ Bestar $^{\circledR}$ SYBR Green qPCR Master Mix, $0.5 \mu \mathrm{l}$ of each primer (miR-150, forward 5'-ACACTCCAG CTGGGTCTCCCAACCCTTGTACC-3' and reverse, 5'-CTC AACTGGTGTCGTGGA-3'; iASPP, forward, 5'-GAAAG CCTGGAACGAGTCTGA-3' and reverse, 5'-GCGCTA GTGAGGTTGTCCT-3'; U6, forward, 5'-CTCGCTTCGG CAGCACA-3' and reverse, 5'-AACGCTTCACGAATTT GCGT-3'; and GAPDH, forward, 5'-TGTTCGTCATGG GTGTGAAC-3' and reverse, 5'-ATGGCATGGACTGTGG TCAT-3'), $1 \mu \mathrm{l}$ cDNA template and $8 \mu \mathrm{l}$ RNase-free $\mathrm{H}_{2} \mathrm{O}$. 

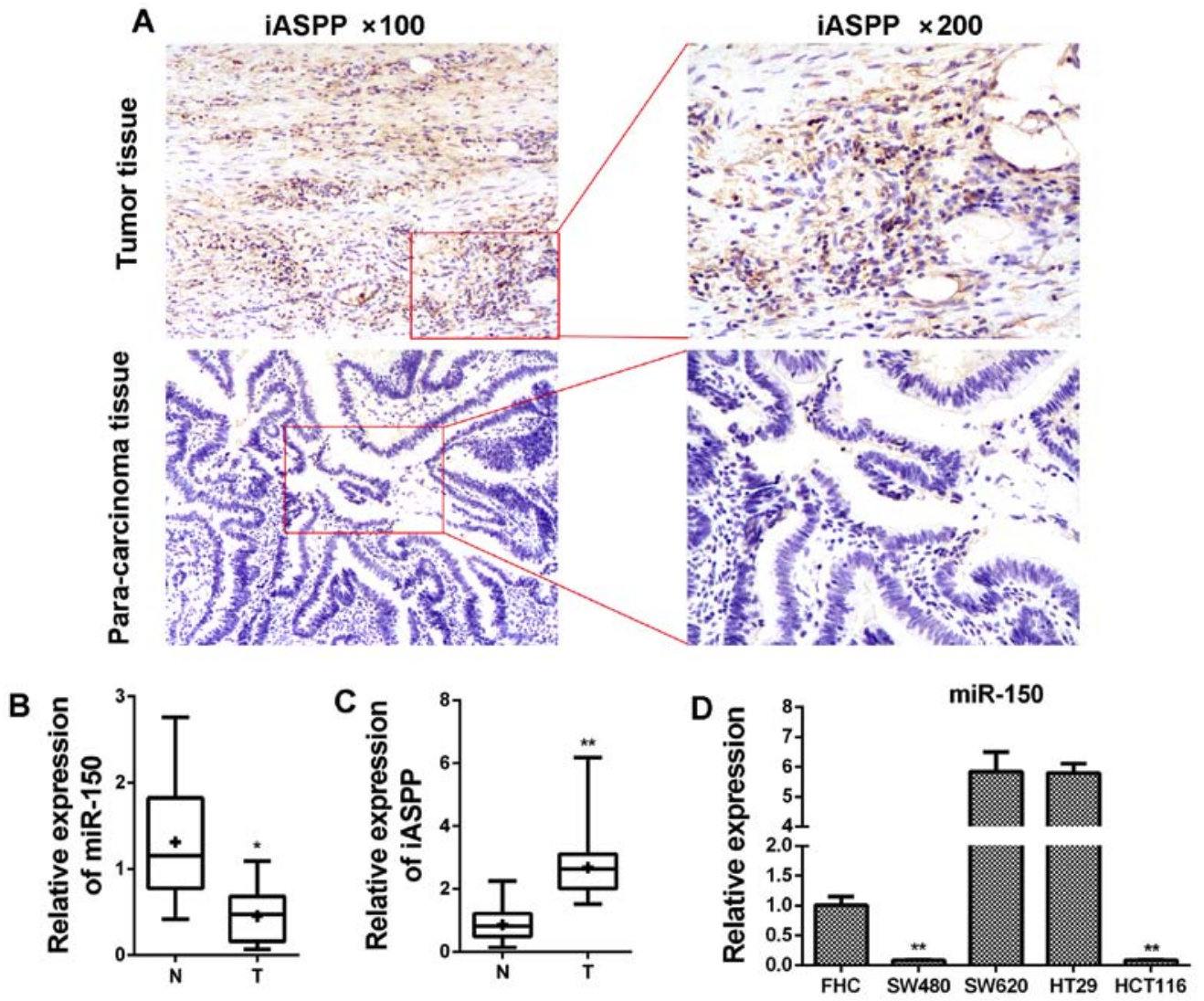

Figure 1. Expression of $m i R-150$ and $i A S P P$ at the mRNA level in 20 pairs of clinical CRC samples and corresponding para-carcinoma samples. (A) The expression of $i A S P P$ was investigated using immunohistochemistry in clinical tissues. (B) miR-150 expression was suppressed in CRC samples. (C) $i A S P P$ was induced in CRC samples. (D) The expression level of miR-150 in human CRC cell lines and FHC cells (colon epithelial cell). N represents para-carcinoma tissues; T represents CRC tumor tissues. ${ }^{*} \mathrm{P}<0.05,{ }^{* *} \mathrm{P}<0.01$ vs. other groups.

Amplification was performed as follows: a denaturation step at $94^{\circ} \mathrm{C}$ for 2 min, followed by 40 cycles of amplification at $94^{\circ} \mathrm{C}$ for $20 \mathrm{sec}, 58^{\circ} \mathrm{C}$ for $20 \mathrm{sec}$ and $72^{\circ} \mathrm{C}$ for $20 \mathrm{sec}$. The reaction was stopped at $25^{\circ} \mathrm{C}$ for $5 \mathrm{~min}$. The relative expression levels were detected and analyzed by Exicycler ${ }^{\mathrm{TM}} 96$ (Bioneer Corp., Daejeon, Korea) based on the formula of $2^{-\Delta \Delta c t}$.

Cell Counting Kit-8 (CCK-8) assay. CCK-8 assay was performed to detect cell viability. Briefly, SW480 cells $\left(1 \times 10^{5}\right.$ cells $\left./ \mathrm{ml}\right)$ that underwent different treatments were seeded into one well of a 96-well plate and incubated for $72 \mathrm{~h}$. Every $24 \mathrm{~h}, 10 \mu \mathrm{l}$ of CCK-8 solution was added to every well and incubated at $37^{\circ} \mathrm{C}$ for a minimum of another $4 \mathrm{~h}$. The OD values were detected at $450 \mathrm{~nm}$ and employed as the representative of cell viability.

Flow cytometry. Cell cycle distribution was determined using flow cytometry. Cells were stained with propidium iodide (PI) in the dark for $20 \mathrm{~min}$ at room temperature. The results were analyzed using a FACS flow cytometer (BD Accuri C6; BD Biosciences, San Jose, CA, USA).

Hoechst staining. DNA damage in cell nuclei was detected using a Hoechst staining method after cells were transfected for 24 h. Cells were stained with Hoechst 33258 (Beyotime Institute of Biotechnology, Haimen, China) according to the manufacturer's instructions.
Scratch assay. To evaluate cell mobility, a scratch assay was performed on the transfected cells. Cells at a density of $2 \times 10^{4}$ cells/well were incubated in one well of a 24 -well plate. After marking reference points, cells were cultured to confluence at $37^{\circ} \mathrm{C}$ for $48 \mathrm{~h}$. Then, a cell-free line was made (regarded to as a scratch), and debris at the edges was removed. Twenty-four hours after the scratch was made, gap distances between the midline were assessed using an optical microscope (Olympus Corp.) in reference to the reference points.

Transwell assays. Transwell assays were performed to detect the mobility of SW480 cells. In brief, cells at a density of $2 \times 10^{4}$ cells/well were incubated in the upper chamber (Corning Costar, Cambridge, MA, USA) after a 24-h serum-free incubation, and the chamber was pre-coated with $40 \mu$ l Matrigel $(0.8 \mu \mathrm{g} / \mu \mathrm{l})$ at $37^{\circ} \mathrm{C}$ for $2 \mathrm{~h}$. Then, the system was placed at $37^{\circ} \mathrm{C}$ for $24 \mathrm{~h}$ and, subsequently, cells in the upper surface were completely removed. The lower surfaces of the chamber were stained for $5 \mathrm{~min}$ using $1 \%(\mathrm{w} / \mathrm{v})$ crystal violet and the cell number was recorded using Image-Pro Plus 6.0 software (Media Cybernetics, Inc., Rockville, MD, USA).

Western blotting. Total cellular protein was extracted using the protein lysate. Western blot analysis was conducted according to Lin et al (22). The membranes were incubated with primary antibodies against iASPP $(1: 4,000)$ and GAPDH $(1: 10,000)$ for $1 \mathrm{~h}$ at room temperature. Secondary HRP-conjugated IgG 
Table I. Characteristics of patients with CRC.

\begin{tabular}{lccc}
\hline & \multicolumn{3}{c}{ Expression of miR-150 } \\
\cline { 2 - 4 } Characteristics & Total & Low & High \\
\hline Age (years) & & & \\
$<60$ & 18 & 10 & 8 \\
$\geq 60$ & 12 & 5 & 7 \\
Sex & & & \\
Male & 17 & 9 & 8 \\
Female & 13 & 6 & 7 \\
Tumor size $(\mathrm{cm})$ & & & \\
$<5$ & 11 & 9 & 13 \\
$\geq 5$ & 19 & 6 & \\
TNM stage & & & 3 \\
I+II & 13 & 10 & 10 \\
III+IV & 17 & 5 & \\
\hline
\end{tabular}

CRC, colorectal cancer; TNM, tumor-node-metastasis.

antibodies $(1: 20,000)$ were then added and incubated for $45 \mathrm{~min}$ at room temperature. The blots were developed and the results were recorded in the Gel Imaging System (ScanWizard Bio; Microtek International, Inc., Taipei, Taiwan). Then, data were analyzed using Image-Pro Plus 6.0 (Media Cybernetics, Inc.).

Statistical analysis. Data were expressed as the mean \pm standard deviation (SD). Student's t-test was performed with a significance level of 0.05. Statistical analyses and graphing were performed using GraphPad Prism 6.01 (GraphPad Software, Inc., Chicago, IL, USA).

\section{Results}

The expression of miR-150 is induced and the expression of iASPP is upregulated in clinical CRC samples. The expression status of $m i R-150$ was determined with $\mathrm{qPCR}$ validation in clinical CRC samples and corresponding para-carcinoma tissues from patients (Table I). As displayed in Fig. 1, the level of $m i R-150$ in CRC samples was lower than that in para-carcinoma samples and the difference was statistically significant (Fig. 1B; $\mathrm{P}<0.05$ ). On the contrary, the expression of $i A S P P$ at the mRNA level was induced in CRC samples (Fig. 1C). Based on the detection in clinical samples, it was inferred that $m i R-150$ had an anti-CRC effect during the progression of the tumor. Furthermore, miR-150 expression was assessed, and lower expression was detected in SW480 and HCT116 cells, and relatively higher expression presented in SW620 and HT29 cells compared with the FHC cell line (Fig. 1D). The expression of iASPP was also investigated using immunohistochemistry in clinical tissues from CRC patients. As displayed in Fig. 1A, the expression of iASPP was relatively higher in tumor tissue than para-carcinoma tissue.

Augmented expression of miR-150 suppresses cell viability and induces cell apoptosis and G1 cell-cycle arrest in SW480

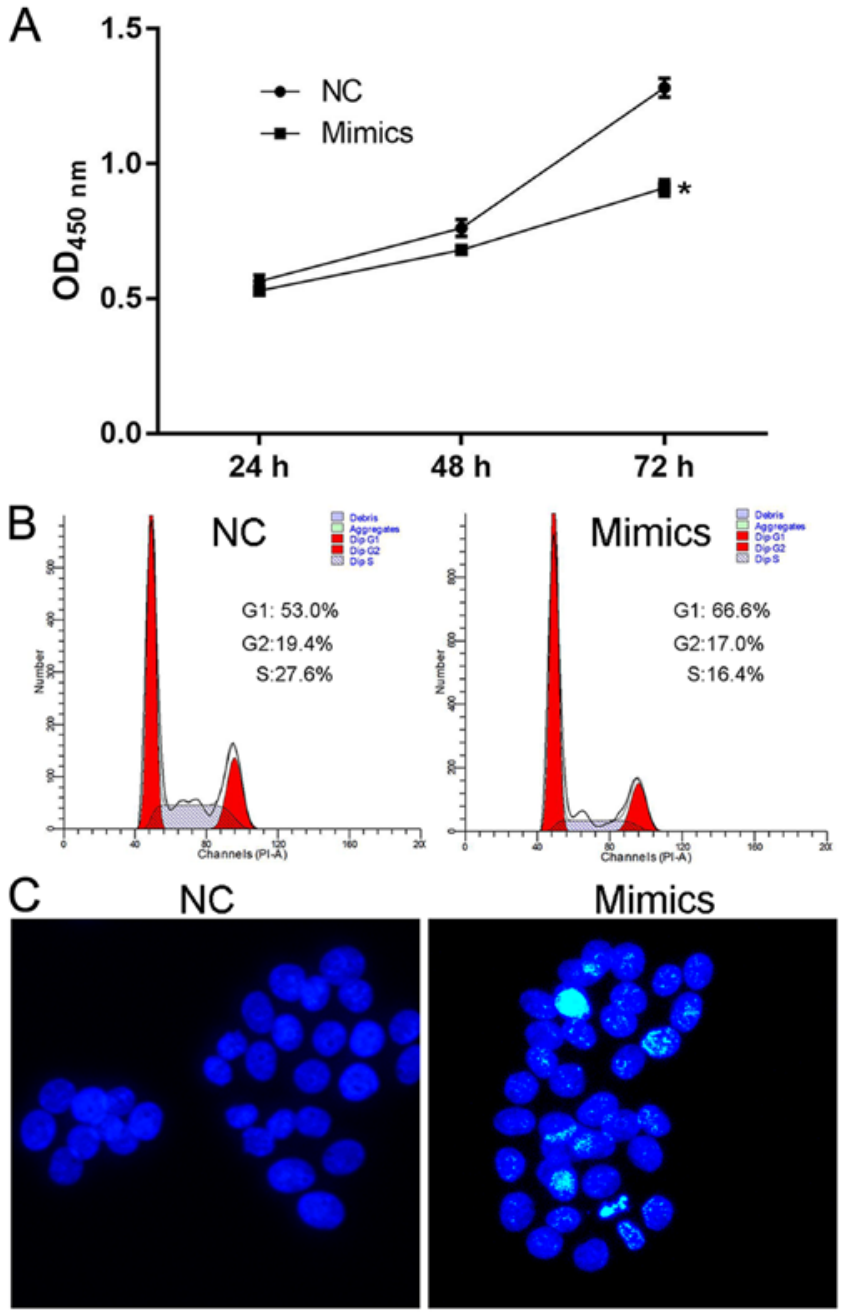

Figure 2. $m i R-150$ decreases cell viability and induces cell cycle arrest and apoptosis in SW480 cells. (A) CCK-8 assay demonstrated that $m i R-150$ decreased the $\mathrm{OD}_{450}$ value at 48 and $72 \mathrm{~h}$. (B) Flow cytometry assay revealed that a higher number of cells distributed in the G1 phase was recorded in the mimics group. (C) Hoechst staining exhibited that DNA damage was observed in the mimics group. NC, negative control; Mimics, $m i R-150$ mimics. ${ }^{*} \mathrm{P}<0.05$ vs. NC.

cells. The effect of $m i R-150$ overexpression on normal cell features was determined to validate the antitumor effect of $\mathrm{miR}$. Being induced by transfection of specific mimics, the upregulated expression of $m i R-150$ significantly decreased the OD450 value in the mimics group at $72 \mathrm{~h}$ (Fig. 2A), representing impaired viability of SW480 cells. Furthermore, the overexpression of $m i R-150$ induced G1 cell cycle arrest and cell apoptosis in the mimics group, as a larger proportion of cells were distributed in the G1 phase (Fig. 2B) and more Hoechst-positive cells (Fig. 2C) were detected in cells transfected with $m i R-150$. Detection focusing on the proliferation potential of SW480 cells confirmed the conclusion that $m i R-150$ is an anti-CRC molecule that influences CRC cells both by decreasing cell proliferation and inducing cell apoptosis.

Augmented expression of miR-150 impairs the migration and invasion ability of SW480 cells. The effect of $m i R-150$ overexpression was further assessed by detecting its effect on the metastasis potential of SW480 cells. For cells subjected to 
A
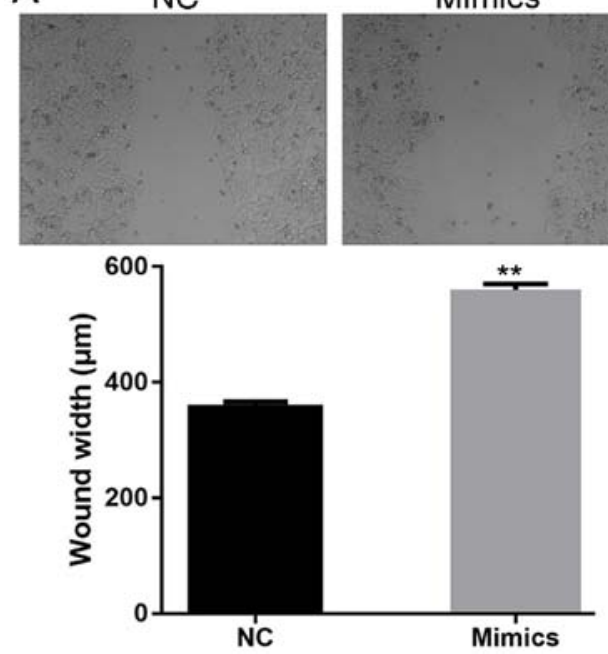

Mimics
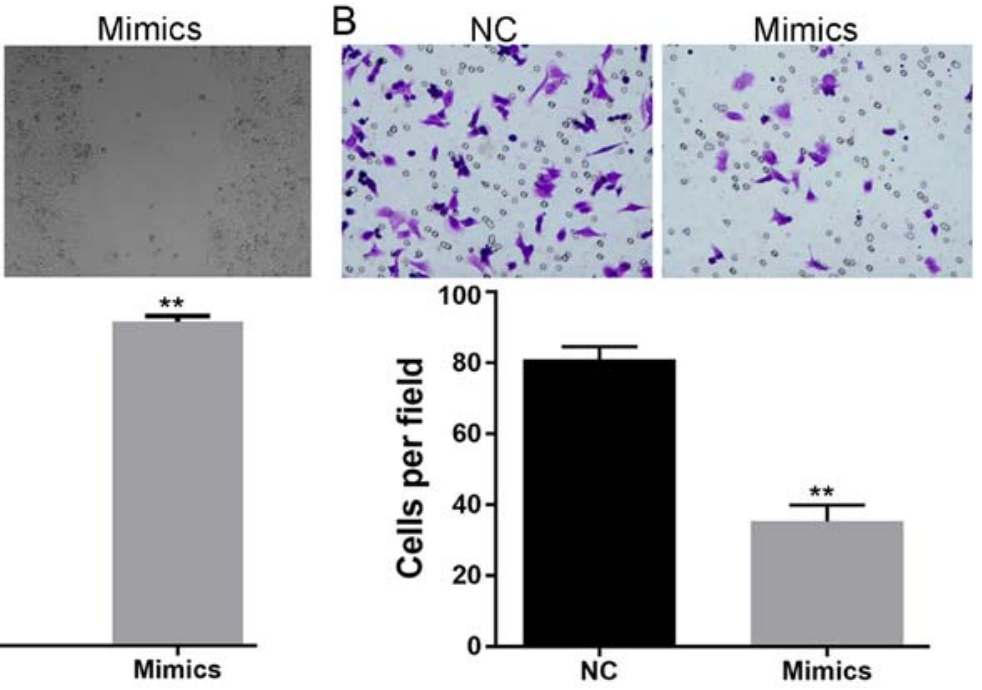

Figure 3. $m i R-150$ inhibits the mobility of SW480 cells. (A) $m i R-150$ mimics resulted in delayed gap closure in SW480 cells. (B) $m i R-150$ mimics resulted in

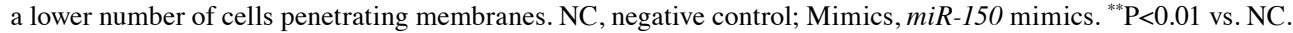

the scratch assay, induced expression of $m i R-150$ resulted in a delayed closure rate of the gap (wider gap width) (Fig. 3A). Furthermore, impaired invasion ability was also detected in SW480 cells with overexpression of $m i R-150$ (Fig. 3B), as less cells penetrating the membranes were recorded in the mimics group. The results of the scratch and Transwell assays together indicated the inhibitory effect of $m i R-150$ on the metastasis potential of CRC cells.

iASPP is directly regulated by miR-150 and plays a promoting role in the oncogenesis of $C R C$. Based on bioinformatic analysis, $i A S P P$ was a potential target of $m i R-150$. In the present study, a possible interaction between the two indicators was determined using a dual-luciferase assay. The results indicated that only cells transfected with $m i R-150$ mimics and wild-type iASPP 3'UTR demonstrated a decreased relative luciferase activity (Fig. 4), which indicated a direct and specific modulating effect of $m i R-150$ on the $i A S P P$ gene.

To confirm the results that iASPP promoted the progression of CRC, the effects of $i A S P P$ knockdown on cell viability, cell apoptosis, cell cycle distribution and cell migration and invasion were also assessed. The expression of iASPP was confirmed by western blot analysis after transfection with siRNA (Fig. 5A). The results indicated that iASPP knockdown exhibited a similar effect on SW480 cells to that of $m i R-150$ overexpression: in the siRNA group, cell viability was decreased (Fig. 5B), G1 cell cycle arrest and apoptosis were induced (Fig. 5C and D) and cell migration and invasion were impaired (Fig. 6).

miR-150 exerts its suppressing effect on CRC cells by targeting $i A S P P$. Given the direct regulating function of $m i R-150$ in the transcription of $i A S P P$, it was hypothesized that the antitumor effect of $m i R-150$ on CRC was dependent on the suppressed function of $i A S P P$. Therefore, the expression of iASPP was induced in $m i R-150$ overexpressed SW480 and HCT116 cells (Figs. 7A and 9A, respectively). Subsequently, the growth and metastasis potential of SW480 and HCT116 cells in different groups were assessed. Based on the results, induced expression
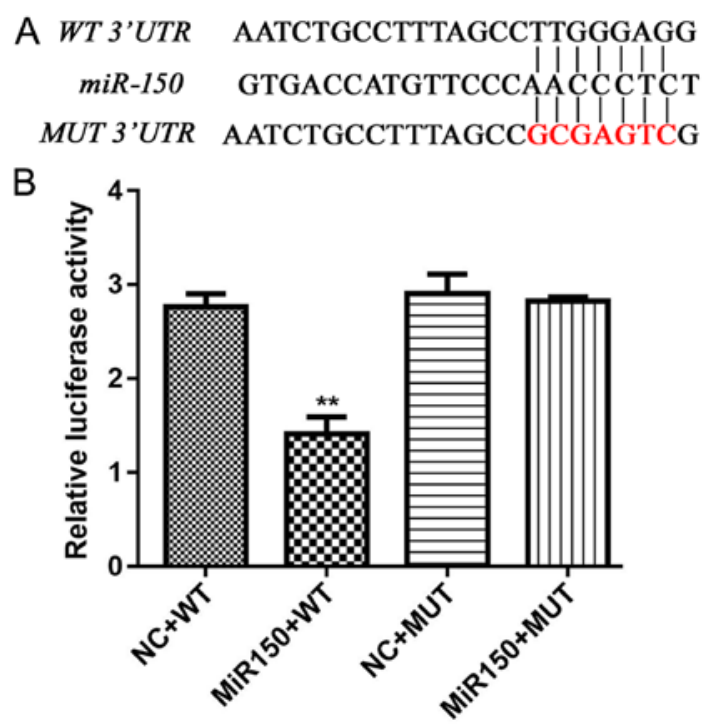

Figure 4. miR-150 directly binds to the 3'UTR of iASPP and suppresses the transcription of the gene. (A) The binding sequence of $m i R-150$ to the 3'UTR of $i A S P P$; $m i R-150$ can bind to the 3'UTR of $i A S P P$ directly. (B) Only cells transfected with $m i R-150$ mimics and wild-type $i A S P P$ 3'UTR demonstrated a decreased relative luciferase activity. ${ }^{* *} \mathrm{P}<0.01$ vs. NC+WT.

of $i A S P P$ counteracted the effect of $m i R-150$ overexpression on SW480 and HCT116 cells. Concerning growth potential, induced iASPP expression increased cell viability, relieved cells from G1 cell cycle arrest and inhibited cell apoptosis in the miRNA+pcDNA group when compared with the miRNA group (Figs. 7B-D and 9B-D). Additionally, upregulated iASPP levels also improved the metastatic potential of SW480 and HCT116 cells, as a faster closure rate and more cells penetrating the membrane were detected in the miRNA+pcDNA group as demonstrated by scratch and Transwell assays (Figs. 8 and 9E-G). It was hypothesized that, without the low level of iASPP, the suppressing function of $m i R-150$ on CRC cells was confounded, which indicated that the anti-CRC function of $m i R-150$ was exerted via the inhibition of $i A S P P$. 

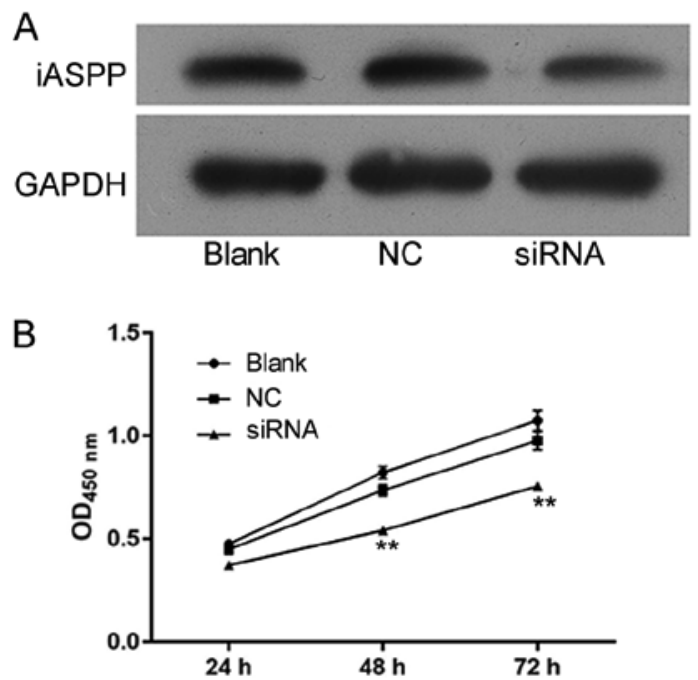

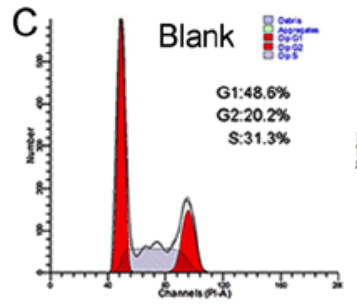

D

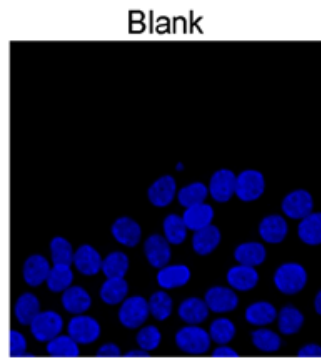

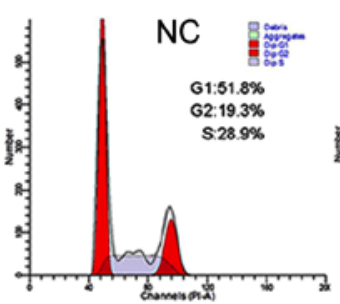
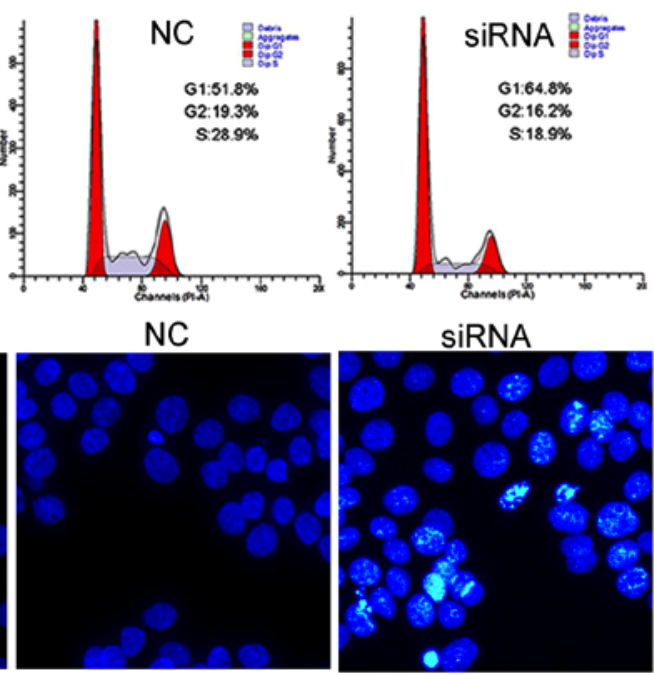

Figure 5. Knockdown of $i A S P P$ decreases cell viability and induces cell cycle arrest and apoptosis in SW480 cells. (A) The expression of iASPP was knocked down by siRNA. (B) CCK-8 assay indicated that iASPP knockdown significantly reduced the OD $\mathrm{OD}_{450}$ value at 48 and $72 \mathrm{~h}$. (C) Flow cytometry assay revealed that a higher number of cells distributed in the G1 phase was recorded in the siRNA group. (D) Hoechst staining revealed that more cells with DNA damage were observed in the siRNA group. Blank, SW480 cells; NC, negative control. ${ }^{* *} \mathrm{P}<0.01 \mathrm{vs.} \mathrm{NC.}$
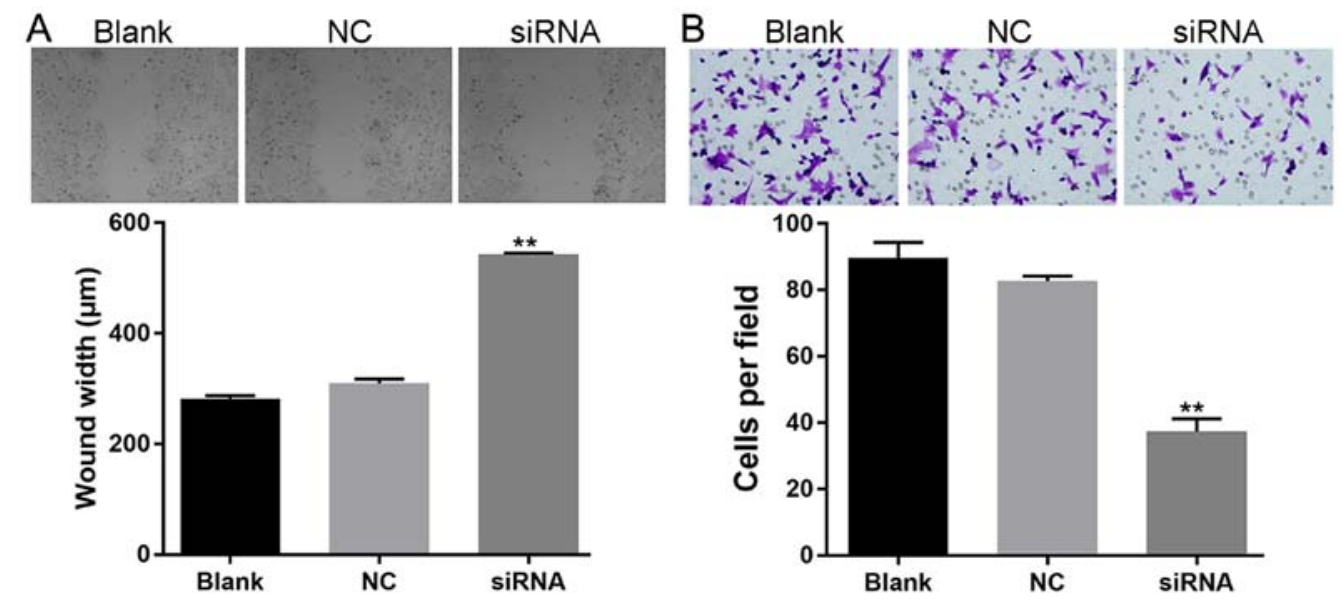

Figure 6. Downregulation of $i$ ASPP inhibits mobility in SW480 cells. (A) Transfection of siRNA resulted in delayed gap closure in SW480 cells. (B) Transfection

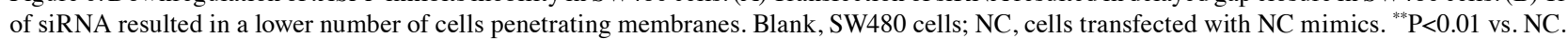

\section{Discussion}

The suppressed expression of miRs in colorectal cancer (CRC) may represent a novel therapeutic avenue for the treatment of CRC (5,23). Among all reported downregulated miRs in CRC, the potential of miR-150 as a biomarker for diagnosing and predicting CRC has been reported in several studies $(7,17)$. In the present study, the data confirmed the decreased level of $m i R-150$ in clinical CRC samples. Furthermore, reintroduction of $m i R-150$ markedly supressed viability, induced apoptosis and inhibited migration and invasion of CRC cells in vitro. The effect of miR-150 on SW480 cells depended on the function of iASPP, the overexpression of which blocked the impairments of miR-150 mimics on SW480 cells. The present study clearly indicated that $m i R-150$ was capable of predicting and suppressing CRC, a finding that deserves further investigation.

Dysregulation of $m i R-150$ has been reported in diverse tumor types. However, the exact function of $m i R-150$ varies by tumor type. For gastric, breast and lung cancer, $m i R-150$ plays a role in promoting the progression of cancer $(13,24,25)$. Conversely, for pancreatic cancer, $m i R-150$ is able to suppress the growth of the tumor by targeting MUC4 (26). The possible involvement of $m i R-150$ in CRC was first reported by Ogata-Kawata et al (27) however, in their study, serum exosomal levels of $m i R-150$ were markedly higher in primary CRC patients than in healthy controls. With emerging attention being paid to the function of $m i R-150$ in CRC, several other researchers have consistently revealed a reduced level of $m i R-150$ in CRC tumors $(5,15,17)$, which indicated that $m i R-150$ is a biomarker associated with CRC prognosis (15). In the present study, qPCR validation of clinical CRC and para-carcinoma samples confirmed the conclusion that $m i R-150$ was suppressed in CRC tissues. Subsequently, induced $m i R-150$ in human CRC cell line SW480 further verified the inhibitory effect of $m i R-150$ on the growth and metastatic potential of CRC cells. To further uncover the 
A

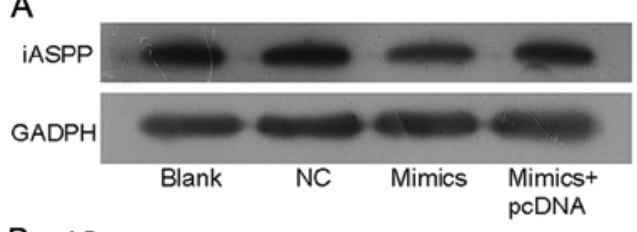

B

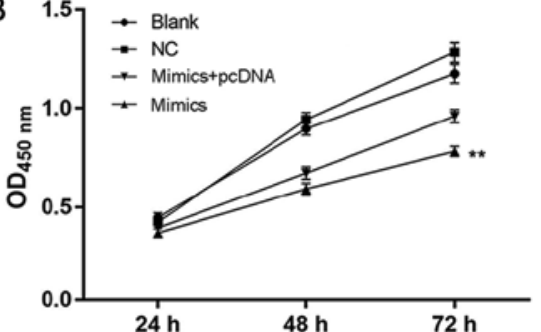

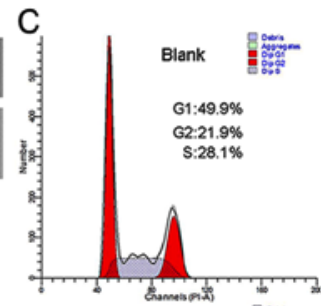

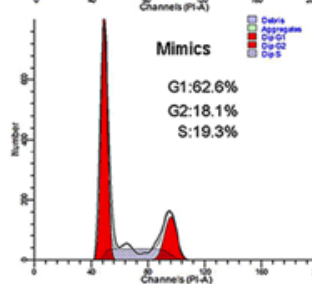

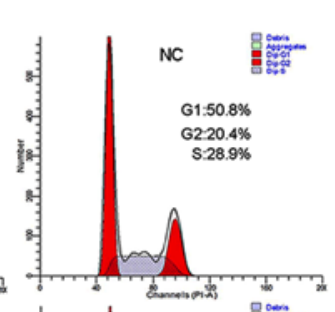

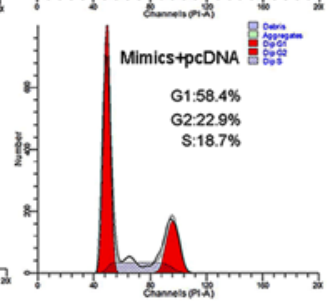

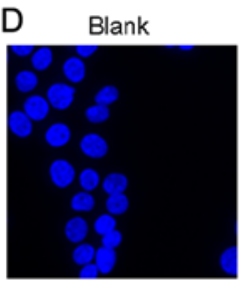

Mimics

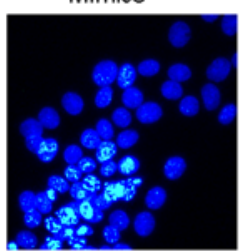

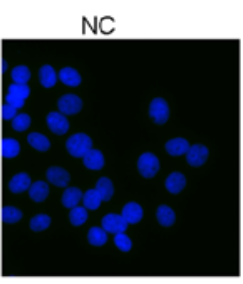

Mimics+pcDNA

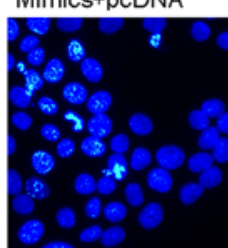

Figure 7. The suppressing effect of $m i R-150$ on CRC cells depends on the overexpression of iASPP. Overexpression of iASPP increases cell viability and inhibits cell cycle arrest and apoptosis in SW480 and HCT116 cells. (A) iASPP was decreased after the cells were transfected with mimics. (B) CCK-8 assay exhibited that downregulation of $i A S P P$ increased the $\mathrm{OD}_{450}$ value at 48 and $72 \mathrm{~h}$ when compared with the mimics group. (C) Flow cytometry assay demonstrated that a lower number of cells distributed in the G1 phase was recorded in the mimics+pcDNA group when compared with the miRNA group. (D) Hoechst staining indicated that less Hoechst-positive cells were observed in the mimics+pcDNA group. Blank, SW480 cells; NC, negative control; Mimics, miR-150 mimics. ${ }^{* *} \mathrm{P}<0.05$ vs. mimics.
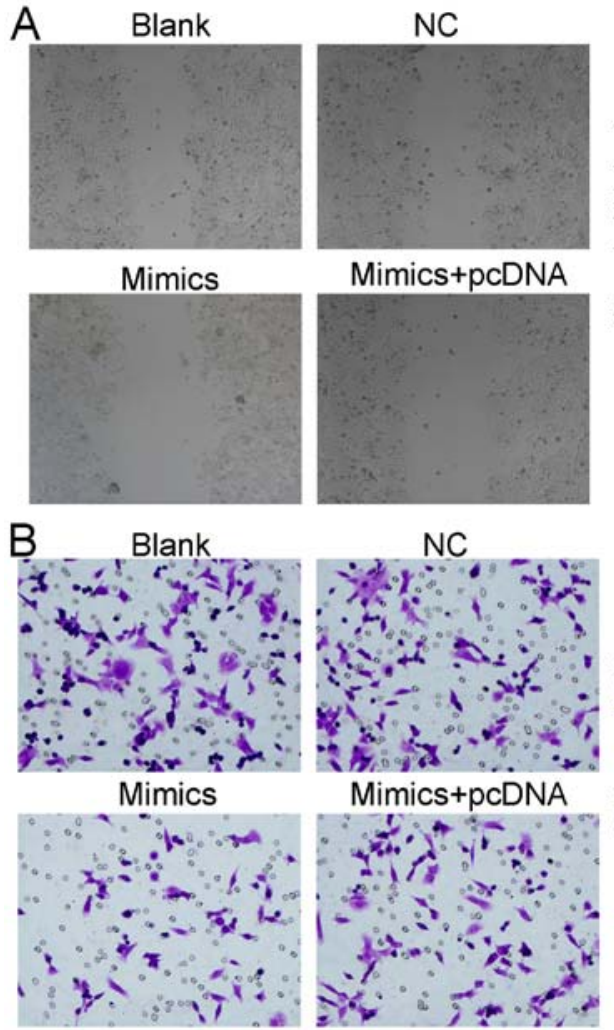
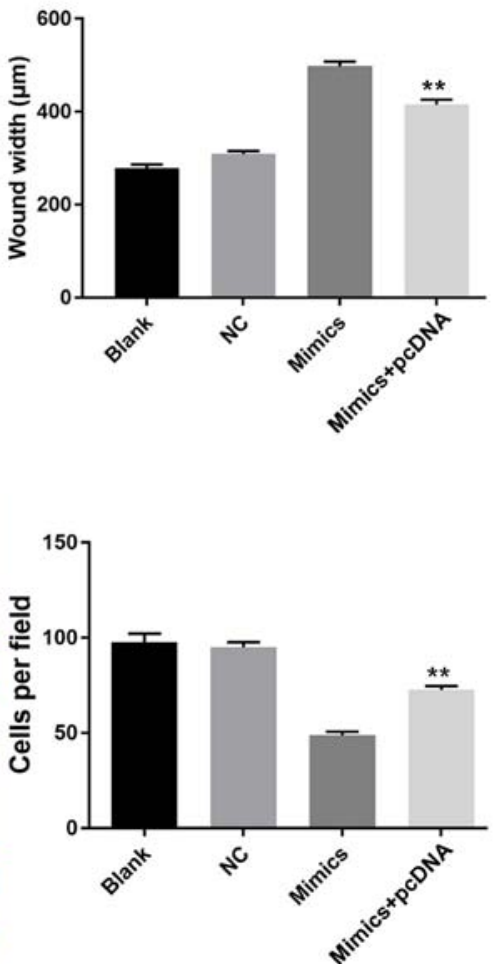

Figure 8. Overexpression of $i A S P P$ increases inhibited mobility in SW480 cell lines. (A) Transfection with the $p c D N A-i A S P P$ vector resulted in a higher gap closure rate in SW480 cells when compared with the mimics group. (B) Transfection with the $p c D N A$ - $i A S P P$ vector resulted in a higher number of cells penetrating membranes. Blank, SW480 cells; NC, negative control; Mimics, $m i R-150$ mimics. ${ }^{* *} \mathrm{P}<0.05$ vs. mimics.

downstream pathways involved in the antagonizing effect of $m i R-150$ on CRC, we also detected the effects of interaction between $m i R-150$ and iASPP on CRC cells.

iASPP acts as a negative regulator of the tumor suppressor p53 and its overexpression has been associated to poor prognosis and survival in some types of cancers $(18,21)$. The gene can suppress apoptosis by deactivating the function of p53 on the promoters of proapoptotic genes (21). In addition to regulating $\mathrm{p} 53$, iASPP has been also proven to inhibit the transcription of RelA/p65 and reduce inflammation (28). Induced expression of miR-124 in CRC cells attenuated cell viability, proliferation and colony formation via inhibition of iASPP protein expression and forced overexpression of iASPP rescued CRC cells from the inhibitory effect of miR-124 (6). Therefore, targeted suppression of iASPP may serve as the mechanism by which its upstream miR prevents oncogenesis 


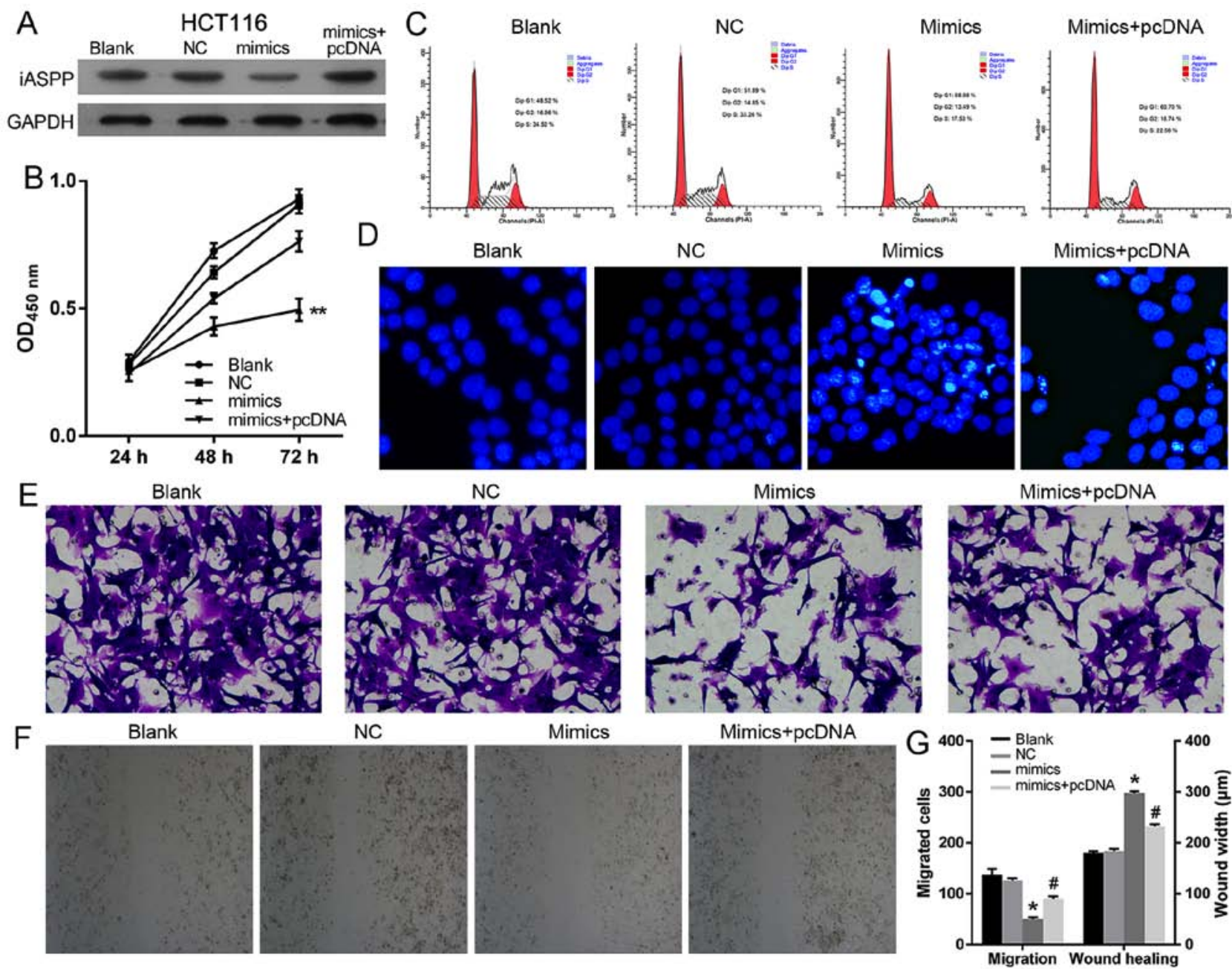

Figure 9. The suppressing effect of $m i R-150$ on HCT116 cells depends on the overexpression of iASPP. (A) The expression of iASPP was assessed after HCT116 cells were transfected with mimics or/and iASPP. (B) Cell proliferation was reversed after the cells were transfected with pcDNA-iASPP compared with the mimics group. (C) Flow cytometry assay indicated that a lower number of cells distributed in the G1 phase was recorded in the mimics+pcDNA group compared with the mimics group. (D) Hoechst staining demonstrated that less Hoechst-positive cells were observed in the mimics+pcDNA group compared with the mimics group. (E-G) The inhibitive effect on cell migration and invasion was reversed in the mimics+pcDNA group compared with the mimics group. Blank, SW480 cells; NC, negative control, Mimics, miR-150 mimics. ${ }^{*} \mathrm{P}<0.05,{ }^{* *} \mathrm{P}<0.01$ vs. $\mathrm{NC},{ }^{*} \mathrm{P}<0.05$ vs. mimics.

of CRC. In the present study, we proved that overexpression of iASPP contributed to the enhanced growth and metastasis of CRC cells. By performing a dual-luciferase assay, iASPP was validated to be a direct target of $m i R-150$ in CRC cells and induced expression of $m i R-150$ restricted the expression of iASPP both at the mRNA and protein levels. However, the impairment of $m i R-150$ in SW480 cells was partially obscured by re-expression of $i A S P P$. Collectively, these results indicated that $m i R-150$ can act as a suppressor on the growth and metastasis of CRC and this effect was exerted by direct inhibition of the expression of iASPP.

In conclusion, the present study provided more evidence supporting the anti-CRC function of miR-150. The low expression $m i R-150$ in clinical samples highlighted the possibility that the molecule may predict poor prognosis in CRC patients. In addition, the suppressed viability, proliferation, migration and invasion in SW480 cells due to enforced expression of $m i R-150$ supported the treatment potential of $m i R-150$ in CRC. The present study also revealed a key role of iASPP in the oncogenesis of CRC, which can be targeted by miR-150. The findings of the present study provided a supplementary mechanism underlying the suppressing effect of $m i R-150$ on $\mathrm{CRC}$ and offered a therapeutic target for future exploration.

\section{Acknowledgements}

Not applicable.

\section{Funding}

The present study was supported by the National Natural Science Foundation of China (no. 61471397).

\section{Availability of data and materials}

All data generated or analyzed during this study are included in this published article.

\section{Authors' contributions}

CL and XD conceived and designed the study. CL and SX performed the experiments. CL and LC wrote the study. CL 
and XD reviewed and edited the manuscript. All authors read and approved the manuscript and agree to be accountable for all aspects of the research in ensuring that the accuracy or integrity of any part of the work are appropriately investigated and resolved.

\section{Ethics approval and consent to participate}

All procedures were approved by the Chinese People's Liberation Army General Hospital from May 2015 to October 2016.

\section{Consent for publication}

Not applicable.

\section{Competing interests}

The authors declare that they have no competing interests.

\section{Reference}

1. McGuire S: World Cancer Report 2014. Geneva, Switzerland: World Health Organization, International Agency for Research on Cancer, WHO Press, 2015. Adv Nutr 7: 418-419, 2016.

2. Siegel RL, Miller KD and Jemal A: Cancer statistics, 2015. CA Cancer J Clin 65: 5-29, 2015.

3. DA Silva FC, Wernhoff P, Dominguez-Barrera $C$ and DominguezValentin M: Update on Hereditary Colorectal Cancer. Anticancer Res 36: 4399-4405, 2016.

4. Strubberg AM and Madison BB: MicroRNAs in the etiology of colorectal cancer: Pathways and clinical implications. Dis Mode Mech 10: 197-214, 2017.

5. Aherne ST, Madden SF, Hughes DJ, Pardini B, Naccarati A, Levy M, Vodicka P, Neary P, Dowling P and Clynes M: Circulating miRNAs miR-34a and miR-150 associated with colorectal cancer progression. BMC Cancer 15: 329, 2015.

6. Liu K,Zhao H, Yao H,Lei S, Lei Z,Li T and Qi H: MicroRNA-124 regulates the proliferation of colorectal cancer cells by targeting iASPP. Biomed Res Int 2013: 867537, 2013.

7. Wang WH, Chen J, Zhao F, Zhang BR, Yu HS, Jin HY and Dai JH: MiR-150-5p suppresses colorectal cancer cell migration and invasion through targeting MUC4. Asian Pac J Cancer Prev 15: 6269-6273, 2014.

8. Bartel DP: MicroRNAs: Genomics, biogenesis, mechanism, and function. Cell 116: 281-297, 2004.

9. Lu J, Getz G, Miska EA, Alvarez-Saavedra E, Lamb J, Peck D, Sweet-Cordero A, Ebert BL, Mak RH, Ferrando AA, et al MicroRNA expression profiles classify human cancers. Nature 435: 834-838, 2005.

10. Volinia S, Calin GA, Liu CG, Ambs S, Cimmino A, Petrocca F, Visone R, Iorio M, Roldo C, Ferracin M, et al: A microRNA expression signature of human solid tumors defines cancer gene targets. Proc Natl Acad Sci USA 103: 2257-2261, 2006.

11. Sarlinova M, Halasa M, Mistuna D, Musak L, Iliev R, Slaby O, Mazuchova J, Valentova V, Plank L and Halasova E: miR-21, miR-221 and miR-150 Are Deregulated in Peripheral Blood of Patients with Colorectal Cancer. Anticancer Res 36: 5449-5454, 2016.

12. Lin YC, Kuo MW, Yu J, Kuo HH, Lin RJ, Lo WL and Yu AL: $\mathrm{c}-\mathrm{Myb}$ is an evolutionary conserved miR-150 target and miR$150 / c-M y b$ interaction is important for embryonic development. Mol Biol Evol 25: 2189-2198, 2008.
13. Wu Q, Jin H, Yang Z, Luo G, Lu Y, Li K, Ren G, Su T, Pan Y, Feng B, et al: MiR-150 promotes gastric cancer proliferation by negatively regulating the pro-apoptotic gene EGR2. Biochem Biophys Res Commun 392: 340-345, 2010.

14. Watanabe A, Tagawa H, Yamashita J, Teshima K, Nara M, Iwamoto K, Kume M, Kameoka Y, Takahashi N, Nakagawa T, et al: The role of microRNA-150 as a tumor suppressor in malignant lymphoma. Leukemia 25: 1324-1334, 2011.

15. Ma Y, Zhang P, Wang F, Zhang H, Yang J, Peng J, Liu W and Qin H: miR-150 as a potential biomarker associated with prognosis and therapeutic outcome in colorectal cancer. Gut 61: 1447-1453, 2012.

16. Pizzini S, Bisognin A, Mandruzzato S, Biasiolo M, Facciolli A, Perilli L, Rossi E, Esposito G, Rugge M, Pilati P, et al: Impact of microRNAs on regulatory networks and pathways in human colorectal carcinogenesis and development of metastasis. BMC Genomics 14: 589, 2013.

17. Feng J, Yang Y, Zhang P, Wang F, Ma Y, Qin H and Wang Y: miR-150 functions as a tumour suppressor in human colorectal cancer by targeting c-Myb. J Cell Mol Med 18: 2125-2134, 2014.

18. Gillotin S: iASPP, a potential drug target in cancer therapy. Leuk Res 33: 1175-1177, 2009.

19. Slee EA, Gillotin S, Bergamaschi D, Royer C, Llanos S, Ali S, Jin $\mathrm{B}$, Trigiante $\mathrm{G}$ and $\mathrm{Lu} \mathrm{X}$ : The $\mathrm{N}$-terminus of a novel isoform of human iASPP is required for its cytoplasmic localization. Oncogene 23: 9007-9016, 2004.

20. Bell HS and Ryan KM: iASPP inhibition: Increased options in targeting the p53 family for cancer therapy. Cancer Res 68: 4959-4962, 2008.

21. Cai Y, Qiu S, Gao X, Gu SZ and Liu ZJ: iASPP inhibits p53-independent apoptosis by inhibiting transcriptional activity of p63/p73 on promoters of proapoptotic genes. Apoptosis 17: 777-783, 2012.

22. Lin W, Zhao Z, Ni Z, Zhao Y, Du W and Chen S: IFI16 restoration in hepatocellular carcinoma induces tumour inhibition via activation of p53 signals and inflammasome. Cell Prolif 50: e12392, 2017

23. Amirkhah R, Schmitz U, Linnebacher M, Wolkenhauer O and Farazmand A: MicroRNA-mRNA interactions in colorectal cancer and their role in tumor progression. Genes Chromosomes Cancer 54: 129-141, 2015.

24. Huang S, Chen Y, Wu W, Ouyang N, Chen J, Li H, Liu X, Su F, Lin L and Yao Y: miR-150 promotes human breast cancer growth and malignant behavior by targeting the pro-apoptotic purinergic P2X7 receptor. PLoS One 8: e80707, 2013.

25. Cao M, Hou D, Liang H, Gong F, Wang Y, Yan X, Jiang X, Wang C, Zhang J, Zen K, et al: miR-150 promotes the proliferation and migration of lung cancer cells by targeting SRC kinase signalling inhibitor 1. Eur J Cancer 50: 1013-1024, 2014.

26. Srivastava SK, Bhardwaj A, Singh S, Arora S, Wang B, Grizzle WE and Singh AP: MicroRNA-150 directly targets MUC4 and suppresses growth and malignant behavior of pancreatic cancer cells. Carcinogenesis 32: 1832-1839, 2011.

27. Ogata-Kawata H, Izumiya M, Kurioka D, Honma Y, Yamada Y, Furuta K, Gunji T, Ohta H, Okamoto H, Sonoda H, et al: Circulating exosomal microRNAs as biomarkers of colon cancer. PLoS One 9: e92921, 2014.

28. Hu Y, Ge W, Wang X, Sutendra G, Zhao K, Dedeić Z, Slee EA, Baer C and $\mathrm{Lu} X$ : Caspase cleavage of iASPP potentiates its ability to inhibit p53 and NF- $\kappa$ B. Oncotarget 6: 42478-42490, 2015.

This work is licensed under a Creative Commons Attribution-NonCommercial-NoDerivatives 4.0 International (CC BY-NC-ND 4.0) License. 